\title{
Supporting Information: Quantifying Triplet State Formation in Zinc Dipyrrin Complexes
}

Norah Z. Alqahtani, Toni G. Blevins, and Catherine E. McCusker*

East Tennessee State University Department of Chemistry, Johnson City, Tennessee 37614

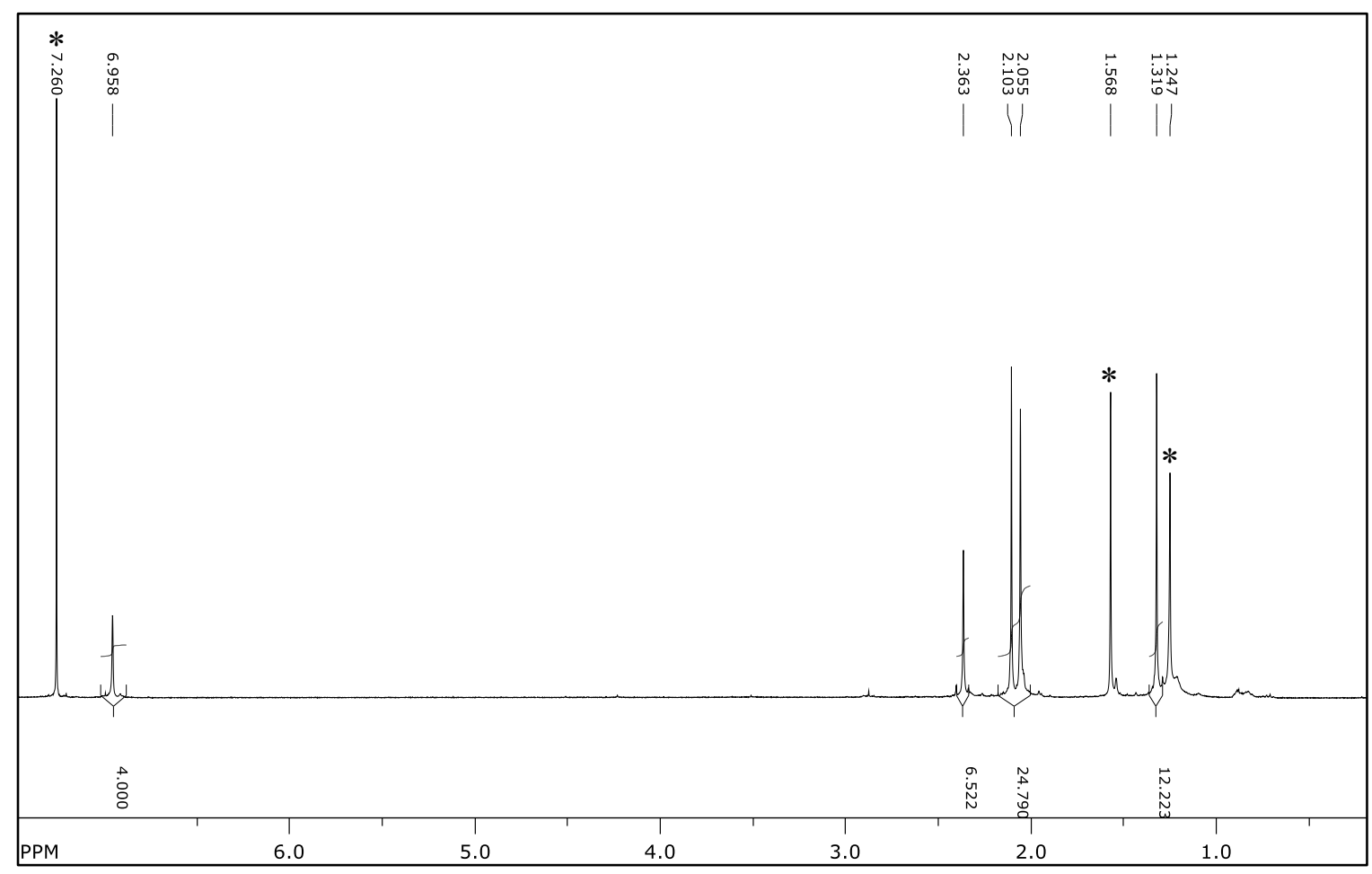

Figure $\mathrm{S} 1 .{ }^{1} \mathrm{H}$ NMR of 2 in $\mathrm{CDCl}_{3}$ Starred peaks are due to water and residual solvent. 


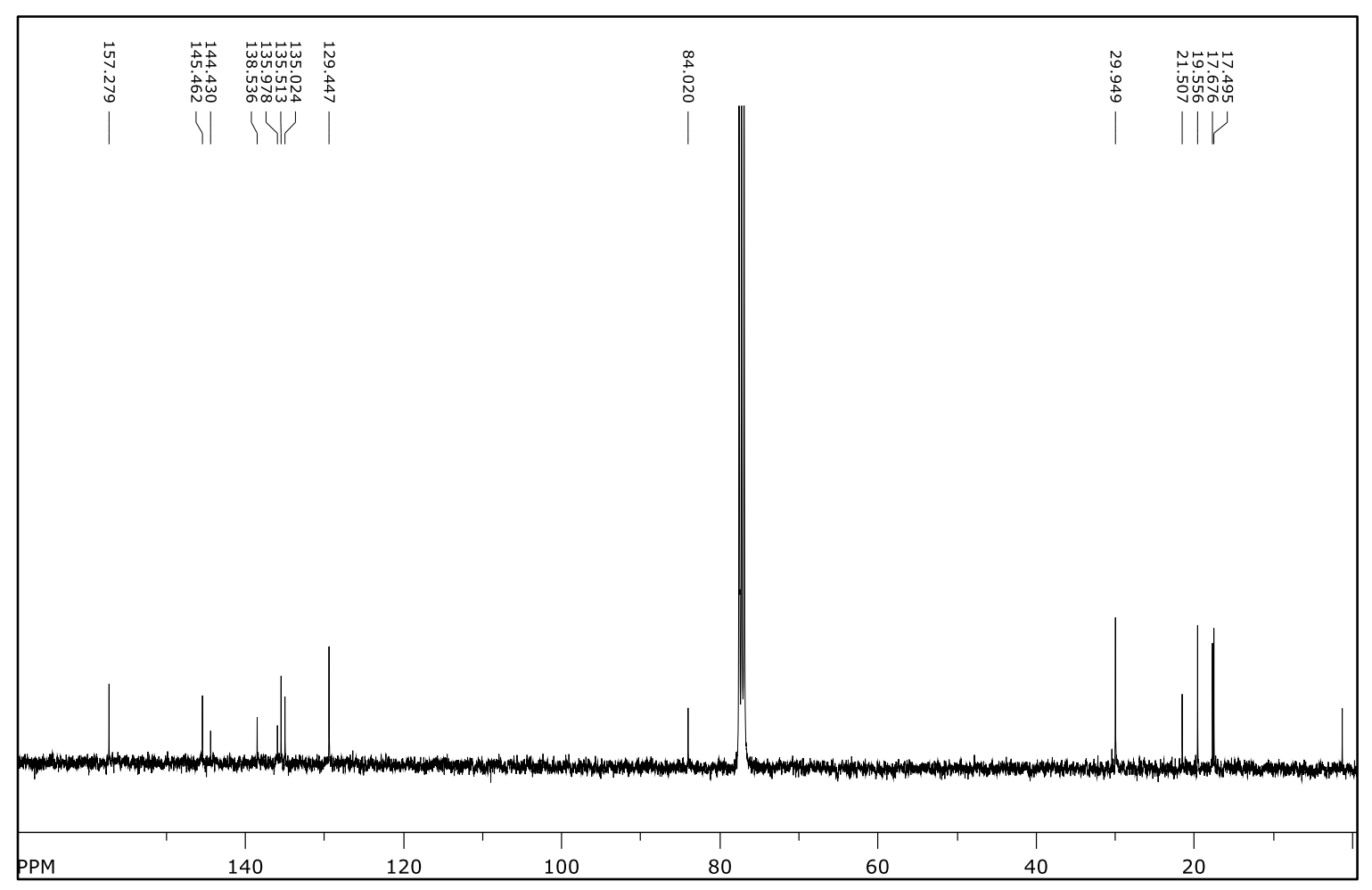

Figure S2. ${ }^{13} \mathrm{C}$ NMR of 2 in $\mathrm{CDCl}_{3}$

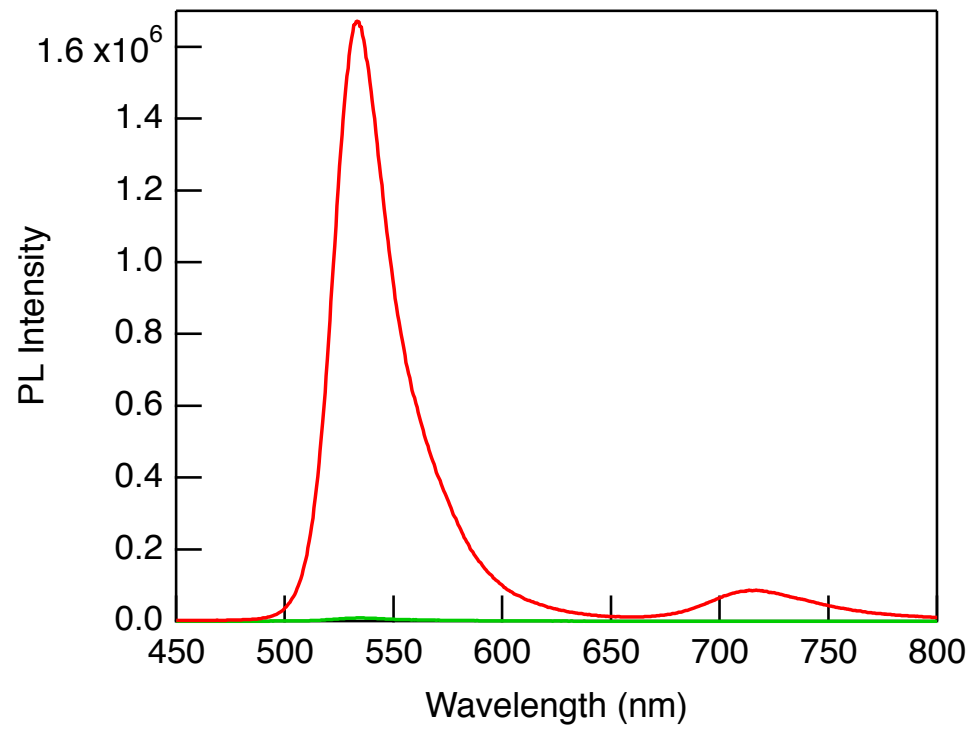

Figure S3. Time gated emission spectra of 2 in deaerated THF showing the prompt (0 ns delay, 50 ns gate width) emission signal in green and the delayed ( $2 \mu \mathrm{s}$ delay, $100 \mu$ sate width) in red 

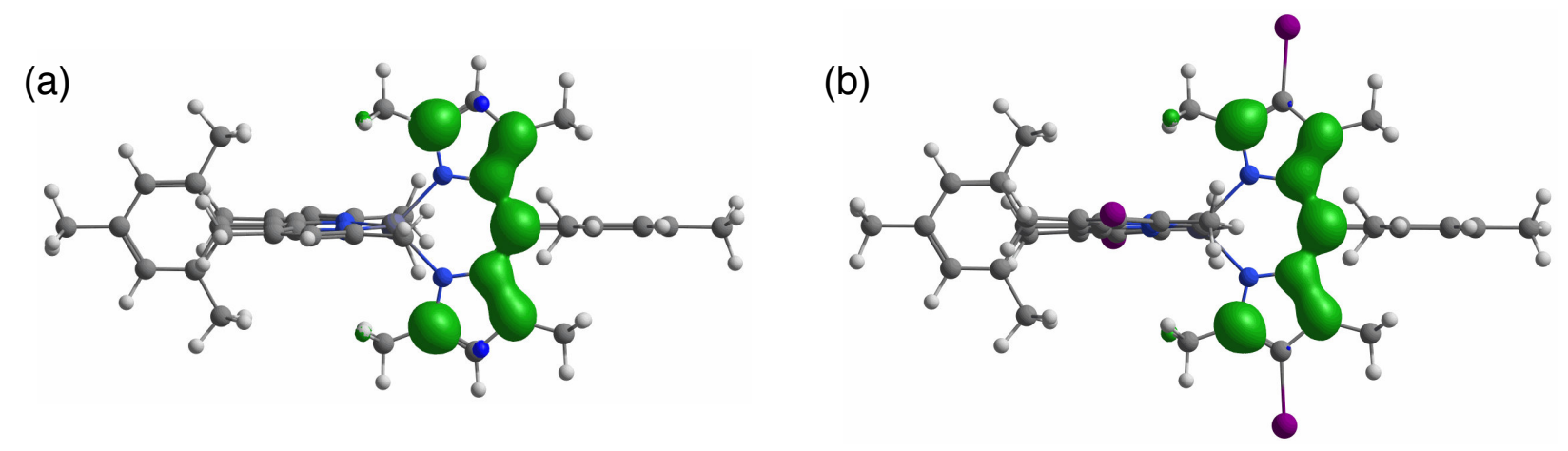

Figure S4. (a) Spin density in the lowest energy triplet state of 1 calculated in a THF continuum. (b) Spin density in the lowest energy triplet state of 2 calculated in a THF continuum.
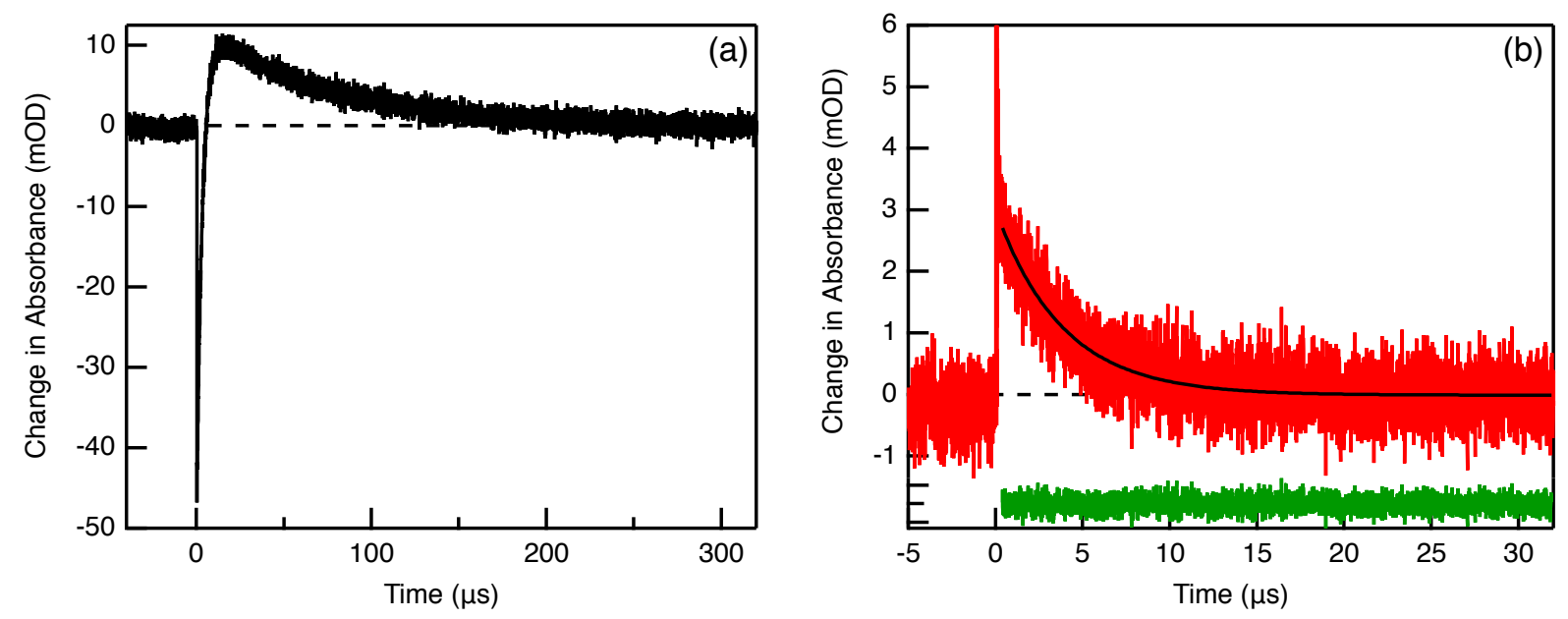

Figure S5. Transient absorption measurements of 1 and perylene $\left(8.0 \times 10^{-5} \mathrm{M}\right)$ in deaerated toluene $\left(\lambda_{e x}=485 \mathrm{~nm}, 1.0 \mathrm{~mJ} /\right.$ pulse). (a) Kinetic decay trace probed at $490 \mathrm{~nm}$. (b) Kinetic decay trace probed at $600 \mathrm{~nm}$. The black line is a single exponential fit $(\tau=3.8 \mu \mathrm{s})$ and the green line is the fit residual. 

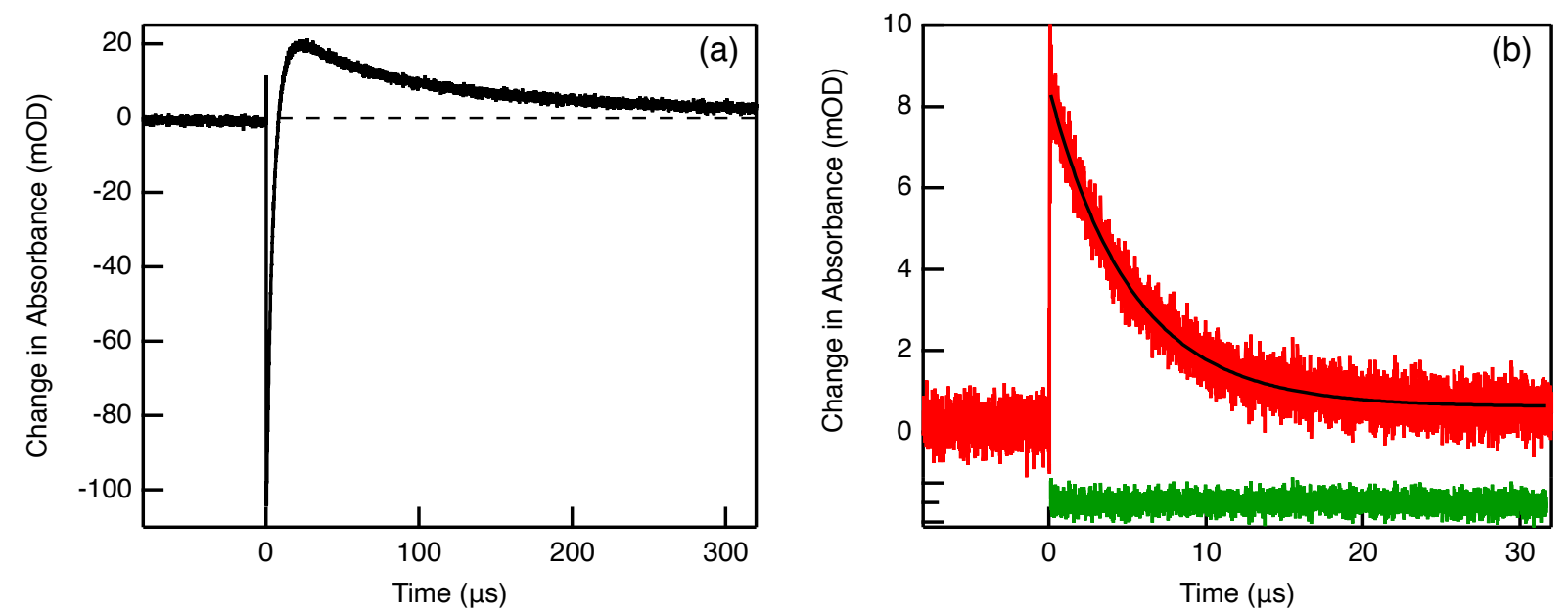

Figure S6. Transient absorption measurements of 1 and perylene $\left(5.5 \times 10^{-5} \mathrm{M}\right)$ in deaerated THF ( $\lambda_{\text {ex }}=485 \mathrm{~nm}, 1.0 \mathrm{~mJ} /$ pulse). (a) Kinetic decay trace probed at $490 \mathrm{~nm}$. (b) Kinetic decay trace probed at $600 \mathrm{~nm}$. The black line is a single exponential fit $(\tau=5.2 \mu \mathrm{s})$ and the green line is the fit residual.
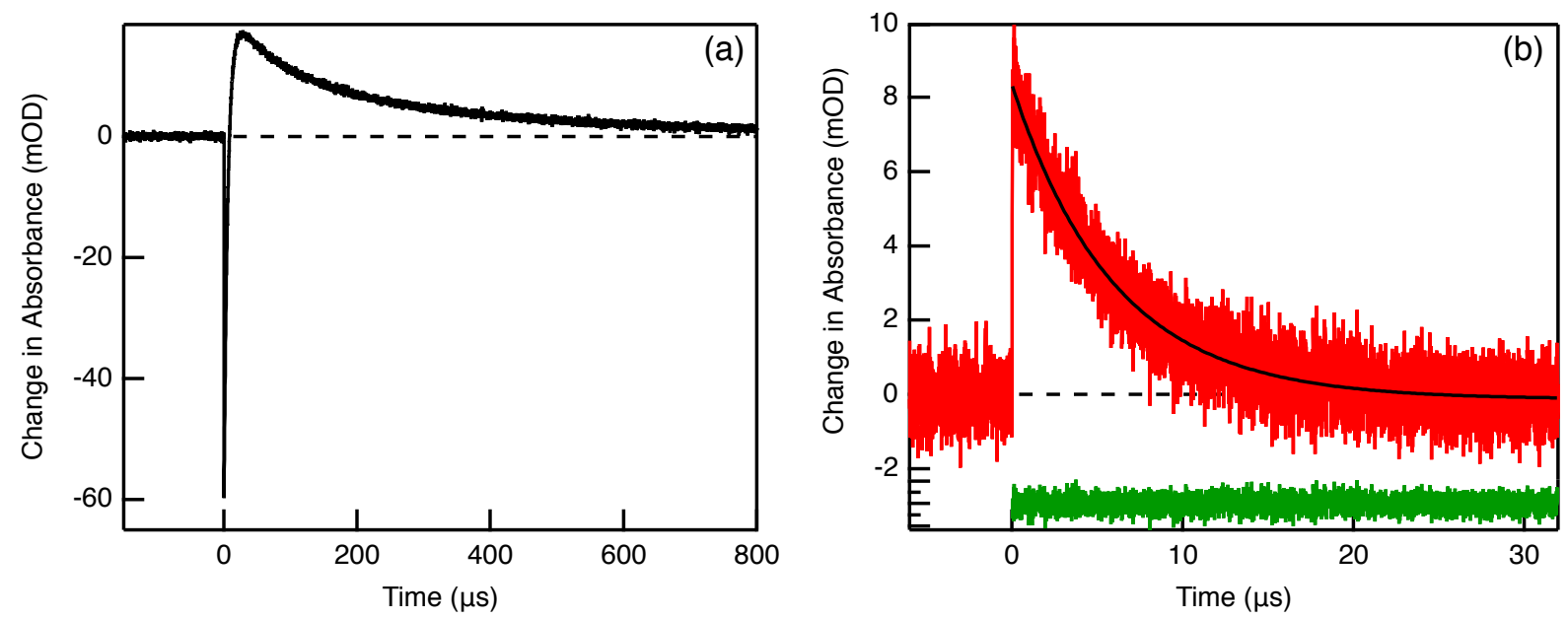

Figure S7. Transient absorption measurements of 2 and perylene $\left(6.7 \times 10^{-5} \mathrm{M}\right)$ in deaerated THF $\left(\lambda_{\text {ex }}=510 \mathrm{~nm}, 1.0 \mathrm{~mJ} /\right.$ pulse). (a) Kinetic decay trace probed at $490 \mathrm{~nm}$. (b) Kinetic decay trace probed at $600 \mathrm{~nm}$. The black line is a single exponential fit $(\tau=6.0 \mu \mathrm{s})$ and the green line is the fit residual. 

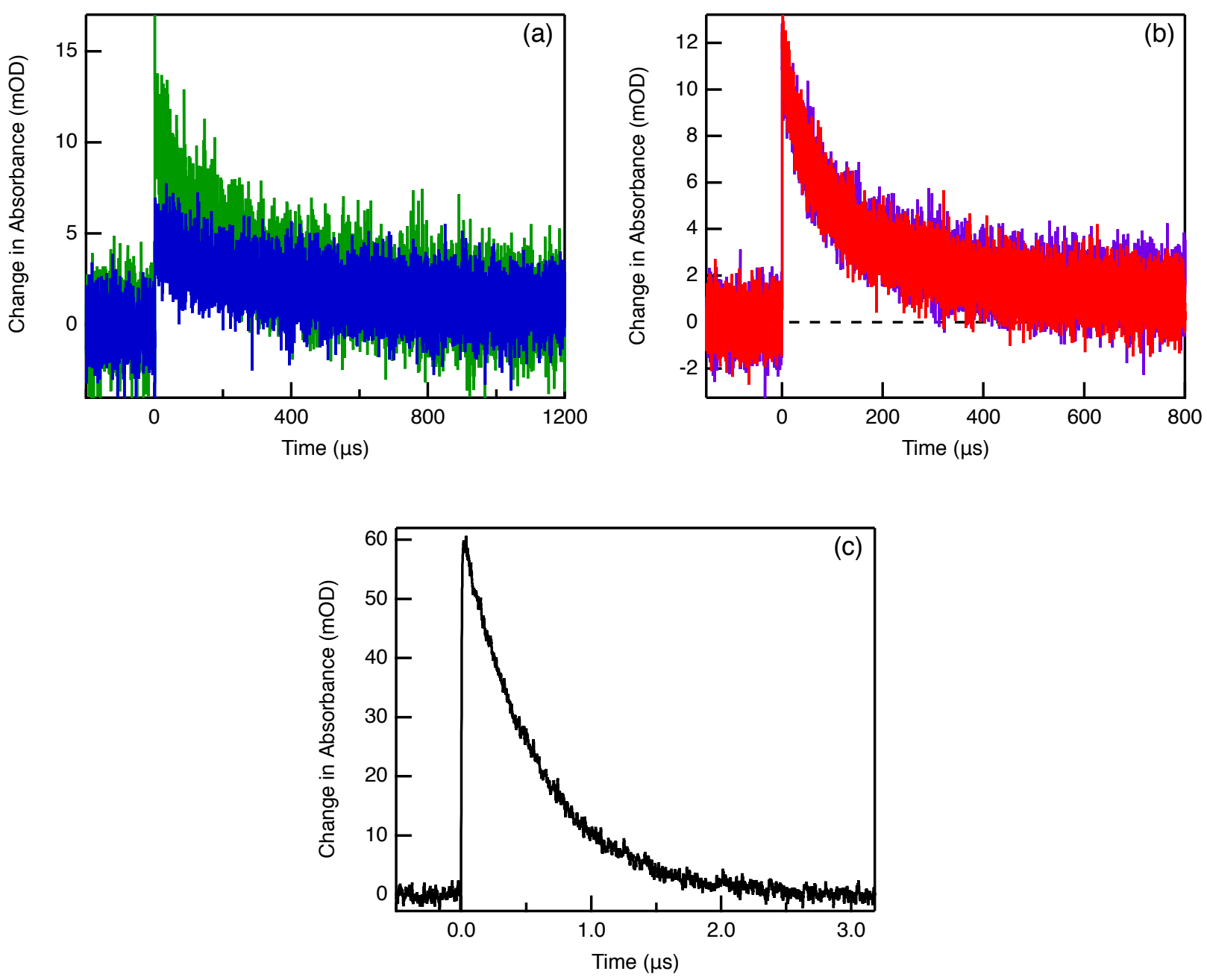

Figure S8. Transient absorption kinetic decay traces ( $\left.\lambda_{\mathrm{ex}}=480 \mathrm{~nm}, 1.0 \mathrm{~mJ} / \mathrm{pulse}\right)$ of (a) 1 in deaerated toluene (blue) and THF (green) probed at $600 \mathrm{~nm}$, (b) 2 in deaerated toluene, (d) 2 in deaerated $\mathrm{THF}$, and (c) $\left[\mathrm{Ru}(\mathrm{bpy})_{3}\right] \mathrm{Cl}_{2}$ in deaerated water. 
Table S1. Optimized triplet state geometry of 1 in toluene continuum

\begin{tabular}{|c|c|c|c|}
\hline C & 1.9102639 & 1.91837305 & -0.87379 \\
\hline $\mathrm{C}$ & 76 & 98 & \\
\hline $\mathrm{N}$ & & & \\
\hline $\mathrm{C}$ & & & \\
\hline C & 916 & 568 & 6855 \\
\hline $\mathrm{C}$ & 787 & 262 & 808 \\
\hline $\mathrm{C}$ & & & \\
\hline C & & & \\
\hline $\mathrm{C}$ & & & 85 \\
\hline C & & 33 & 08 \\
\hline $\mathrm{N}$ & 21 & 32 & \\
\hline C & 94 & & \\
\hline C & & & \\
\hline C & & & 589 \\
\hline $\mathrm{C}$ & & & \\
\hline $\mathrm{C}$ & & 2 & 81 \\
\hline $\mathrm{C}$ & 8 & 95 & 952 \\
\hline $\mathrm{H}$ & 45 & 56 & 6383 \\
\hline C & 08 & 29 & 937 \\
\hline $\mathrm{C}$ & & 7 & 18 \\
\hline C & & & \\
\hline $\mathrm{H}$ & & & 45 \\
\hline C & & & 803 \\
\hline C & & & 1851 \\
\hline C & 86 & & 675 \\
\hline $\mathrm{Zn}$ & & 3.4 & 336 \\
\hline C & 05 & & 84 \\
\hline $\mathrm{H}$ & & & 92 \\
\hline $\mathrm{H}$ & 97 & 7 & \\
\hline $\mathrm{H}$ & & & 943 \\
\hline $\mathrm{H}$ & 25 & 23 & 4456 \\
\hline $\mathrm{H}$ & 27 & 3.3 & 574 \\
\hline $\mathrm{H}$ & 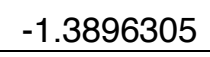 & 10 & 362 \\
\hline $\mathrm{H}$ & & 3 & 4.0 \\
\hline $\mathrm{H}$ & -4.0321308 & -1.4969567 & 4.04044599 \\
\hline $\mathrm{H}$ & 5953 & 0.063308 & 75459 \\
\hline $\mathrm{H}$ & 1.68765825 & 2.05541474 & 8716 \\
\hline $\mathrm{H}$ & 3.15 & 33 & -3 \\
\hline $\mathrm{H}$ & 33 & 3. & -3 \\
\hline
\end{tabular}

S6 


\begin{tabular}{|c|c|c|c|}
\hline $\mathrm{H}$ & .231661 & -0.6901624 & 0.504 \\
\hline $\mathrm{H}$ & 0.297104 & 79 & \\
\hline $\mathrm{H}$ & & & 66 \\
\hline $\mathrm{H}$ & & & \\
\hline $\mathrm{H}$ & & & \\
\hline $\mathrm{H}$ & & & \\
\hline $\mathrm{H}$ & & & \\
\hline $\mathrm{H}$ & & 5 & \\
\hline $\mathrm{H}$ & & 28 & 865 \\
\hline C & 1 & 98 & 83 \\
\hline $\mathrm{C}$ & & & \\
\hline $\mathrm{N}$ & & & \\
\hline $\mathrm{C}$ & & & 64 \\
\hline $\mathrm{C}$ & 5 & & 76 \\
\hline C & 34 & & 031 \\
\hline $\mathrm{C}$ & 82 & & 183 \\
\hline C & & & \\
\hline $\mathrm{C}$ & & & \\
\hline C & & & \\
\hline $\mathrm{N}$ & & & \\
\hline C & & & \\
\hline $\mathrm{C}$ & 86 & & 313 \\
\hline C & 1832792 & 36 & 73 \\
\hline $\mathrm{C}$ & & 8 & \\
\hline C & & & \\
\hline $\mathrm{C}$ & & & \\
\hline $\mathrm{H}$ & 1 & 1 & \\
\hline C & 39 & 38 & 356 \\
\hline $\mathrm{C}$ & 5 & 25 & 31 \\
\hline C & & & \\
\hline $\mathrm{H}$ & & & \\
\hline C & 1 & 31 & 38 \\
\hline $\mathrm{C}$ & 1 & 242 & 302 \\
\hline C & 0. & & \\
\hline $\mathrm{C}$ & & 2 & 92 \\
\hline $\mathrm{H}$ & -2 & 4 & 5 \\
\hline $\mathrm{H}$ & -2.6803234 & 5.39468622 & 01050710 \\
\hline $\mathrm{H}$ & 0 & 6.97253072 & -9.014427 \\
\hline $\mathrm{H}$ & 2.27272498 & 3.61908367 & -7.6286596 \\
\hline $\mathrm{H}$ & 2.70237025 & 5.09466103 & -6.7592041 \\
\hline
\end{tabular}




\begin{tabular}{|l|r|r|r|}
\hline$H$ & 3.52988576 & 4.69798231 & -8.2880313 \\
\hline$H$ & 2.14917826 & 8.10332988 & -11.682104 \\
\hline$H$ & 0.46344472 & 7.9903774 & -12.249058 \\
\hline$H$ & 1.60799612 & 6.66155836 & -12.557238 \\
\hline$H$ & 0.12670817 & 8.26024285 & -7.2572652 \\
\hline$H$ & 1.77407088 & 7.66579071 & -7.0761768 \\
\hline$H$ & 1.18727262 & 8.9698795 & -6.0180478 \\
\hline$H$ & -2.4468797 & -0.2890199 & -5.7769454 \\
\hline$H$ & -2.5951308 & 0.90562091 & -4.45414 \\
\hline$H$ & -1.068757 & 0.03960483 & -4.6998562 \\
\hline$H$ & 0.4741149 & 3.23520802 & -9.6843402 \\
\hline$H$ & -1.1742411 & 3.82646968 & -9.8682488 \\
\hline$H$ & -0.8333724 & 2.09210361 & -10.068905 \\
\hline$H$ & -1.5743024 & 0.63151001 & -8.181529 \\
\hline$H$ & -5.9286151 & 4.0678749 & -2.1240226 \\
\hline$H$ & 0.18365889 & 6.87703705 & -1.4038794 \\
\hline$H$ & 0.01792111 & 5.1050376 & -1.5811888 \\
\hline$H$ & -1.4083069 & 6.14685224 & -1.7213162 \\
\hline$H$ & 2.61650666 & 0.65697326 & -1.3706379 \\
\hline$H$ & 0.68417655 & 8.29801541 & -3.66467 \\
\hline & & & \\
\hline
\end{tabular}

Table S2. Optimized triplet state geometry of 1 in THF continuum.

\begin{tabular}{|l|r|r|r|}
\hline C & -1.9245551 & 1.90296587 & -0.8854178 \\
\hline C & -0.5459955 & 1.83040224 & -1.2940909 \\
\hline $\mathrm{N}$ & -0.0294609 & 2.40751649 & -2.4276234 \\
\hline $\mathrm{C}$ & 1.3177076 & 2.14035104 & -2.4832085 \\
\hline $\mathrm{C}$ & 1.70099258 & 1.36927226 & -1.367347 \\
\hline $\mathrm{C}$ & 0.56923512 & 1.14489628 & -0.5912576 \\
\hline $\mathrm{C}$ & -2.9657122 & 2.56105786 & -1.6308719 \\
\hline $\mathrm{C}$ & -4.39649 & 2.69962155 & -1.2546263 \\
\hline $\mathrm{C}$ & -4.9963216 & 3.39815461 & -2.2966665 \\
\hline $\mathrm{C}$ & -4.0055667 & 3.68083991 & -3.2584474 \\
\hline $\mathrm{N}$ & -2.7952206 & 3.17624751 & -2.8465007 \\
\hline $\mathrm{C}$ & -5.1358763 & 2.23626731 & -0.0360808 \\
\hline $\mathrm{C}$ & -4.152203 & 4.40922871 & -4.5503255 \\
\hline $\mathrm{C}$ & -2.3032552 & 1.24493412 & 0.41246687 \\
\hline $\mathrm{C}$ & -2.7620767 & -0.0916287 & 0.42855813 \\
\hline $\mathrm{C}$ & -2.8808892 & -0.8825686 & -0.8533665 \\
\hline $\mathrm{C}$ & -3.112403 & -0.688409 & 1.65020283 \\
\hline & & & \\
\hline
\end{tabular}




\begin{tabular}{|c|c|c|c|}
\hline $\mathrm{H}$ & 316 & .7240151 & 1.65 \\
\hline C & & 8 & \\
\hline $\mathrm{C}$ & & & \\
\hline C & & & 2 \\
\hline $\mathrm{H}$ & & & \\
\hline C & & & \\
\hline $\mathrm{C}$ & & & \\
\hline C & & & \\
\hline $\mathrm{Zn}$ & 83 & 44 & \\
\hline $\mathrm{C}$ & 5 & 6 & 98 \\
\hline $\mathrm{H}$ & & & \\
\hline $\mathrm{H}$ & & & \\
\hline $\mathrm{H}$ & & 1 & 45 \\
\hline $\mathrm{H}$ & & 02 & 939 \\
\hline $\mathrm{H}$ & 11 & & 679 \\
\hline $\mathrm{H}$ & & & 322 \\
\hline $\mathrm{H}$ & & & \\
\hline $\mathrm{H}$ & & & \\
\hline $\mathrm{H}$ & & & \\
\hline $\mathrm{H}$ & 22 & & 18 \\
\hline $\mathrm{H}$ & & & 533 \\
\hline $\mathrm{H}$ & 775 & & 039 \\
\hline $\mathrm{H}$ & 65 & & 082 \\
\hline $\mathrm{H}$ & & & \\
\hline $\mathrm{H}$ & & & \\
\hline $\mathrm{H}$ & & & \\
\hline $\mathrm{H}$ & 06 & 4 & 324 \\
\hline $\mathrm{H}$ & 97 & & \\
\hline $\mathrm{H}$ & 1 & 57 & 03 \\
\hline $\mathrm{H}$ & & & \\
\hline $\mathrm{H}$ & & & \\
\hline C & 7 & 0 & 48 \\
\hline C & 97 & 2 & 368 \\
\hline $\mathrm{N}$ & 31 & 3 & \\
\hline $\mathrm{C}$ & & & \\
\hline $\mathrm{C}$ & 965 & 2 & 21 \\
\hline $\mathrm{C}$ & & 4 & -5.5022532 \\
\hline $\mathrm{C}$ & 03 & 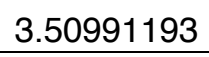 & -6.834649 \\
\hline C & -0.6152061 & 2.64570086 & -8.0038635 \\
\hline $\mathrm{C}$ & -1.0256008 & 1.40061759 & -7.5397272 \\
\hline
\end{tabular}




\begin{tabular}{|c|c|c|c|}
\hline $\mathrm{C}$ & .2161927 & 458 & -0.1305 \\
\hline $\mathrm{N}$ & 99 & & \\
\hline $\mathrm{C}$ & & & \\
\hline C & & & \\
\hline C & & & \\
\hline C & & & \\
\hline $\mathrm{C}$ & & & \\
\hline C & & & \\
\hline $\mathrm{H}$ & & & \\
\hline $\mathrm{C}$ & 9 & & 19 \\
\hline $\mathrm{C}$ & & & \\
\hline $\mathrm{C}$ & & & \\
\hline $\mathrm{H}$ & 1 & & 73 \\
\hline $\mathrm{C}$ & 92 & 3 & 629 \\
\hline C & & & 159 \\
\hline $\mathrm{C}$ & & & 338 \\
\hline C & & & \\
\hline $\mathrm{H}$ & & & \\
\hline $\mathrm{H}$ & & & \\
\hline $\mathrm{H}$ & & & \\
\hline $\mathrm{H}$ & & & 668 \\
\hline $\mathrm{H}$ & & & 634 \\
\hline $\mathrm{H}$ & & 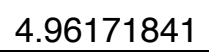 & 63 \\
\hline $\mathrm{H}$ & 2.15239621 & & \\
\hline $\mathrm{H}$ & & & \\
\hline $\mathrm{H}$ & & & \\
\hline $\mathrm{H}$ & & 0 & \\
\hline $\mathrm{H}$ & & & 521 \\
\hline $\mathrm{H}$ & 8 & 9. & 491 \\
\hline $\mathrm{H}$ & & 32 & 47 \\
\hline H & & & \\
\hline $\mathrm{H}$ & 4 & 0 & 77 \\
\hline $\mathrm{H}$ & 05 & 3.2 & -9 \\
\hline $\mathrm{H}$ & 94 & 9 & 558 \\
\hline $\mathrm{H}$ & & & \\
\hline $\mathrm{H}$ & 091 & 5 & -8.143646 \\
\hline $\mathrm{H}$ & 0 & $y$ & \\
\hline$\Pi$ & 02 & 6.99957359 & \\
\hline $\mathrm{H}$ & 423 & $5.2167 / 183$ & -1.5855233 \\
\hline $\mathrm{H}$ & -1.7218316 & 6.14609887 & -1.807821 \\
\hline
\end{tabular}

S10 


\begin{tabular}{|l|l|l|l|}
\hline$H$ & 2.70871609 & 1.01115037 & -1.1560366 \\
\hline$H$ & 0.27924164 & 8.41267356 & -3.7104279 \\
\hline
\end{tabular}

Table S3. Optimized triplet state geometry of $\mathbf{2}$ in toluene continuum.

\begin{tabular}{|l|r|r|r|}
\hline $\mathrm{C}$ & -1.3233698 & 2.02199017 & -0.6358786 \\
\hline $\mathrm{C}$ & -0.4117036 & 1.26598784 & -1.4251754 \\
\hline $\mathrm{N}$ & 0.21284436 & 1.75874685 & -2.5802446 \\
\hline $\mathrm{C}$ & 1.02079166 & 0.8160739 & -3.0751259 \\
\hline $\mathrm{C}$ & 0.94351398 & -0.3404695 & -2.251642 \\
\hline $\mathrm{C}$ & 0.0548574 & -0.0951289 & -1.206169 \\
\hline $\mathrm{C}$ & -1.7882257 & 3.34473046 & -0.8806216 \\
\hline $\mathrm{C}$ & -2.6990257 & 4.14280133 & -0.0733045 \\
\hline $\mathrm{C}$ & -2.835261 & 5.34264083 & -0.7695766 \\
\hline $\mathrm{C}$ & -2.0447666 & 5.28558992 & -1.9500507 \\
\hline $\mathrm{N}$ & -1.4266813 & 4.10182518 & -2.0044312 \\
\hline $\mathrm{C}$ & -3.3836946 & 3.83870643 & 1.22567625 \\
\hline $\mathrm{C}$ & -1.8760945 & 6.34780087 & -2.9877888 \\
\hline $\mathrm{C}$ & -1.8553667 & 1.34603828 & 0.59952216 \\
\hline $\mathrm{C}$ & -3.0598847 & 0.60853202 & 0.53589279 \\
\hline $\mathrm{C}$ & -3.8311343 & 0.48130628 & -0.7573569 \\
\hline $\mathrm{C}$ & -3.5356182 & -0.0131061 & 1.69725397 \\
\hline $\mathrm{H}$ & -4.4677277 & -0.5848934 & 1.64389733 \\
\hline $\mathrm{C}$ & -2.8550373 & 0.07555077 & 2.92094977 \\
\hline $\mathrm{C}$ & -3.3973549 & -0.6061591 & 4.15440157 \\
\hline $\mathrm{C}$ & -1.6655176 & 0.81302061 & 2.95646325 \\
\hline $\mathrm{H}$ & -1.116823 & 0.89770696 & 3.89962186 \\
\hline $\mathrm{C}$ & -1.1504709 & 1.45063895 & 1.81696337 \\
\hline $\mathrm{C}$ & 0.13780962 & 2.23490046 & 1.91228907 \\
\hline $\mathrm{C}$ & -0.2840852 & -1.0895764 & -0.1361839 \\
\hline $\mathrm{Zn}$ & -0.1154676 & 3.5519917 & -3.4236281 \\
\hline $\mathrm{C}$ & 1.83750235 & 1.00174311 & -4.3129284 \\
\hline $\mathrm{H}$ & -3.218125 & 0.0201838 & -1.5492792 \\
\hline $\mathrm{H}$ & -4.1459605 & 1.46664196 & -1.1387325 \\
\hline $\mathrm{H}$ & -4.7319326 & -0.1348974 & -0.6220231 \\
\hline $\mathrm{H}$ & -0.0071109 & 3.28753546 & 1.6181853 \\
\hline $\mathrm{H}$ & 0.91084578 & 1.8258529 & 1.24097672 \\
\hline 0.53543473 & 2.21970752 & 2.93751908 \\
\hline $\mathrm{H}$ & -4.4032495 & -0.2307394 & 4.40810253 \\
\hline
\end{tabular}




\begin{tabular}{|c|c|c|c|}
\hline $\mathrm{H}$ & .7460749 & 2035 & 5.0261970 \\
\hline $\mathrm{H}$ & & & \\
\hline $\mathrm{H}$ & & & \\
\hline $\mathrm{H}$ & & & \\
\hline $\mathrm{H}$ & & & \\
\hline $\mathrm{H}$ & & & \\
\hline $\mathrm{H}$ & & & \\
\hline $\mathrm{H}$ & & & \\
\hline $\mathrm{H}$ & & & \\
\hline $\mathrm{H}$ & 5 & & 21 \\
\hline $\mathrm{H}$ & & & \\
\hline $\mathrm{H}$ & & & \\
\hline $\mathrm{H}$ & & & 33 \\
\hline $\mathrm{C}$ & 9 & 78 & 12 \\
\hline C & & & 174 \\
\hline $\mathrm{N}$ & & & \\
\hline C & & & \\
\hline $\mathrm{C}$ & & & \\
\hline C & & & \\
\hline $\mathrm{C}$ & & & \\
\hline C & & & \\
\hline $\mathrm{C}$ & & & 965 \\
\hline C & 4 & 4 & \\
\hline $\mathrm{N}$ & & & \\
\hline C & & & \\
\hline $\mathrm{C}$ & & & \\
\hline $\mathrm{C}$ & 2 & 6 & \\
\hline $\mathrm{C}$ & & 02 & 795 \\
\hline $\mathrm{C}$ & 9 & 33 & 59 \\
\hline C & & & \\
\hline $\mathrm{H}$ & & & \\
\hline C & 2.651181 & 3 & 23 \\
\hline $\mathrm{C}$ & 67 & 346 & -1 \\
\hline C & 69 & 5.8 & \\
\hline $\mathrm{H}$ & & & 68 \\
\hline C & 2.69142032 & 4 & 4 \\
\hline $\mathrm{C}$ & 3.29219659 & 3 & \\
\hline $\mathrm{C}$ & (c) & 1 & -5.6934538 \\
\hline 1 & 76248 & 1.06958465 & -2.321039 \\
\hline $\mathrm{C}$ & 2.19956836 & 4.72432405 & -1.3946717 \\
\hline
\end{tabular}

S12 


\begin{tabular}{|l|r|r|r|}
\hline $\mathrm{H}$ & 0.26322524 & 7.84511849 & -6.0595371 \\
\hline $\mathrm{H}$ & -0.9210881 & 7.02006634 & -7.0743494 \\
\hline $\mathrm{H}$ & -0.3078384 & 8.62124478 & -7.5602926 \\
\hline $\mathrm{H}$ & 2.52982243 & 3.06342007 & -7.7202688 \\
\hline $\mathrm{H}$ & 3.73329673 & 3.9212823 & -6.75645 \\
\hline $\mathrm{H}$ & 4.08107314 & 3.53692547 & -8.4614508 \\
\hline $\mathrm{H}$ & 3.58214904 & 8.73898423 & -10.7675 \\
\hline $\mathrm{H}$ & 2.38499633 & 7.88909823 & -11.758249 \\
\hline $\mathrm{H}$ & 3.98697001 & 7.15932259 & -11.48429 \\
\hline $\mathrm{H}$ & 2.98390817 & 7.84287804 & -6.1928914 \\
\hline $\mathrm{H}$ & 4.16883321 & 6.57653973 & -6.4954563 \\
\hline $\mathrm{H}$ & 4.42180111 & 7.71412942 & -5.1523655 \\
\hline $\mathrm{H}$ & -3.5443617 & 1.75870346 & -5.4864822 \\
\hline $\mathrm{H}$ & -2.9705193 & 2.7011972 & -4.0765907 \\
\hline $\mathrm{H}$ & -2.1213405 & 1.22241917 & -4.5558773 \\
\hline $\mathrm{H}$ & 0.57676132 & 4.0274149 & -9.3370071 \\
\hline $\mathrm{H}$ & -0.5323735 & 5.38781451 & -9.2022221 \\
\hline $\mathrm{H}$ & -1.1443233 & 3.80377946 & -9.7299926 \\
\hline $\mathrm{I}$ & -3.2661732 & 2.21544578 & -8.494894 \\
\hline $\mathrm{I}$ & -3.9976315 & 7.01743105 & -0.2201617 \\
\hline $\mathrm{H}$ & 3.06660809 & 5.03701367 & -0.7983488 \\
\hline $\mathrm{H}$ & 2.11039479 & 3.62626888 & -1.346749 \\
\hline $\mathrm{H}$ & 1.29068158 & 5.13729401 & -0.9200834 \\
\hline $\mathrm{I}$ & 2.02765621 & -2.1140323 & -2.6215344 \\
\hline & & & \\
\hline
\end{tabular}

Table S4. Optimized triplet state geometry of 2 in THF continuum.

\begin{tabular}{|l|r|r|r|}
\hline $\mathrm{C}$ & -0.0381851 & 1.87808441 & 0.21850879 \\
\hline $\mathrm{C}$ & 0.25053214 & 1.20035519 & -1.0207603 \\
\hline $\mathrm{N}$ & 1.09635383 & 1.6755454 & -1.9967203 \\
\hline $\mathrm{C}$ & 1.13141241 & 0.78588793 & -3.0357954 \\
\hline $\mathrm{C}$ & 0.28316875 & -0.3079303 & -2.7297833 \\
\hline $\mathrm{C}$ & -0.2931678 & -0.1012054 & -1.4783863 \\
\hline $\mathrm{C}$ & 0.53184033 & 3.14233456 & 0.61169932 \\
\hline $\mathrm{C}$ & 0.27043043 & 3.89257253 & 1.86372739 \\
\hline $\mathrm{C}$ & 1.06250464 & 5.03459424 & 1.76700911 \\
\hline $\mathrm{C}$ & 1.7700789 & 5.00504017 & 0.53903605 \\
\hline $\mathrm{N}$ & 1.43490899 & 3.86301057 & -0.1359561 \\
\hline $\mathrm{C}$ & -0.6293484 & 3.56111424 & 3.01214545 \\
\hline $\mathrm{C}$ & 2.72571375 & 5.99352581 & -0.0303355 \\
\hline
\end{tabular}




\begin{tabular}{|c|c|c|c|}
\hline $\mathrm{C}$ & 201 & 1.22035231 & 1. \\
\hline C & 27 & & 100 \\
\hline $\mathrm{C}$ & & & \\
\hline C & & & \\
\hline $\mathrm{H}$ & & & \\
\hline C & & & \\
\hline $\mathrm{C}$ & & & \\
\hline C & & & \\
\hline $\mathrm{H}$ & 91 & & 39 \\
\hline $\mathrm{C}$ & & 0.3 & 79 \\
\hline $\mathrm{C}$ & & & \\
\hline $\mathrm{C}$ & & & \\
\hline $\mathrm{Zn}$ & & & 35 \\
\hline $\mathrm{C}$ & 9 & 8 & 395 \\
\hline $\mathrm{H}$ & & & 305 \\
\hline $\mathrm{H}$ & & & 336 \\
\hline $\mathrm{H}$ & & & \\
\hline $\mathrm{H}$ & & & \\
\hline $\mathrm{H}$ & & & \\
\hline $\mathrm{H}$ & & & \\
\hline $\mathrm{H}$ & & & 994 \\
\hline $\mathrm{H}$ & & & 112 \\
\hline $\mathrm{H}$ & & & 34 \\
\hline $\mathrm{H}$ & & & \\
\hline $\mathrm{H}$ & & & \\
\hline $\mathrm{H}$ & & & \\
\hline $\mathrm{H}$ & & & \\
\hline $\mathrm{H}$ & 8 & & \\
\hline $\mathrm{H}$ & 3 & & 38 \\
\hline $\mathrm{H}$ & & 6.796 & 08 \\
\hline $\mathrm{H}$ & & & \\
\hline $\mathrm{H}$ & 3.67076504 & $.50 \angle 0<11$ & 44 \\
\hline $\mathrm{H}$ & 9 & 1 & 572 \\
\hline $\mathrm{H}$ & 4 & 3. & 266 \\
\hline $\mathrm{H}$ & & 33 & 62 \\
\hline $\mathrm{C}$ & 63 & 1 & 3 \\
\hline $\mathrm{C}$ & 4.89140269 & 3 & -3.1632998 \\
\hline $\mathrm{N}$ & 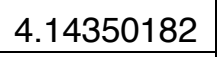 & 000 & 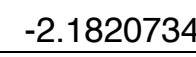 \\
\hline C & 96625 & 2.4881269 & -1.4616115 \\
\hline $\mathrm{C}$ & 2861413 & 2.62454868 & -1.9592231 \\
\hline
\end{tabular}

S14 


\begin{tabular}{|c|c|c|c|}
\hline $\mathrm{C}$ & 471 & .5204346 & -3.06 \\
\hline $\mathrm{C}$ & 59 & & \\
\hline C & & & \\
\hline C & & & \\
\hline $\mathrm{C}$ & & & \\
\hline $\mathrm{N}$ & & & \\
\hline $\mathrm{C}$ & & & \\
\hline C & & & \\
\hline $\mathrm{C}$ & 93 & 54 & \\
\hline C & 9 & & 03 \\
\hline $\mathrm{C}$ & & & \\
\hline $\mathrm{C}$ & & & \\
\hline $\mathrm{H}$ & & & 89 \\
\hline $\mathrm{C}$ & & & 249 \\
\hline C & & & 002 \\
\hline $\mathrm{C}$ & & & \\
\hline $\mathrm{H}$ & & & \\
\hline $\mathrm{C}$ & & & \\
\hline C & & & \\
\hline $\mathrm{C}$ & & & \\
\hline I & & & 555 \\
\hline $\mathrm{C}$ & 732 & & 438 \\
\hline $\mathrm{H}$ & 62 & & 81 \\
\hline $\mathrm{H}$ & 8 & & \\
\hline $\mathrm{H}$ & 6.27004639 & & \\
\hline $\mathrm{H}$ & & & \\
\hline $\mathrm{H}$ & 69 & & 385 \\
\hline $\mathrm{H}$ & & & 272 \\
\hline $\mathrm{H}$ & 84 & 4 & 416 \\
\hline $\mathrm{H}$ & & & \\
\hline 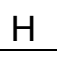 & & & \\
\hline 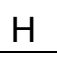 & 3 & ( & 94 \\
\hline $\mathrm{H}$ & 86 & 1 & 343 \\
\hline $\mathrm{H}$ & 8.3 & 3.3 & \\
\hline $\mathrm{H}$ & & & 96 \\
\hline $\mathrm{H}$ & 9 & 3 & -2.6857905 \\
\hline $\mathrm{H}$ & -0.4402498 & 4.08055311 & \\
\hline$\pi$ & 3 & 6.43529148 & \\
\hline $\mathrm{H}$ & 7692637 & 1.1089695 & -5.8009927 \\
\hline 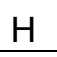 & 2.23994783 & 7.64463643 & -6.7046101 \\
\hline
\end{tabular}

S15 


\begin{tabular}{|l|r|r|r|}
\hline I & -0.5082118 & 7.27487382 & -5.81984 \\
\hline I & 1.21628398 & 6.58854191 & 3.18578523 \\
\hline H & 5.03060183 & 1.90669224 & 0.60720505 \\
\hline$H$ & 4.72100731 & 0.57164264 & -0.5140584 \\
\hline H & 3.42413532 & 1.74356275 & -0.1517314 \\
\hline I & -0.0194701 & -1.9641682 & -4.001296 \\
\hline
\end{tabular}

S16 